\title{
Una nuova lettura di P.Oxy. 2248
}

\author{
Daniela Immacolata Cagnazzo \\ (University of Bari Aldo Moro)
}

\section{A new reading of $P .0 x y .2248$}

\begin{abstract}

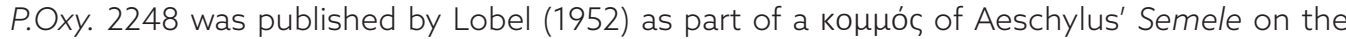
basis of a palaeographical standpoint (cf. P.Oxy. 2164). According to modern scholars, the Aeschylean authorship of the papyrus could likely be confirmed thanks to some textual expressions (cf. II. 2, 5, 12). In fact, the context seems to suggest a dialogue between the Chorus and another character about an adverse fate. The text, however, can be generally reconstructed, though not in detail, and from the reconstruction a more or less clear picture of its contents may be presented. There are still uncertain readings and some unresolved issues in the text, but it is my hope that this paper could overcome some of these difficulties.
\end{abstract}

\section{Keywords}

Aeschylus; Greek papyri; ancient Greek drama; Fragmenta dubia

Vorrei ringraziare gli anonimi refeers per i suggerimenti dispensatimi, e Fabio Frisino per aver letto le bozze del lavoro. La traduzione di ogni passo è stata da me svolta. Una riproduzione fotografica ad alta definizione del papiro è consultabile al seguente link: http://163.1.169.40/gsdl/collect/POxy/index/assoc/HASHa38f. dir/POxy.v0020.n2248.a.01.lores.jpg. 
P.Oxy. $2248\left(\mathrm{MP}^{3} 40=\mathrm{LDAB} 115=\mathrm{TM} 59020\right)$ ha un'estensione di $2.3 \times 7 \mathrm{~cm}$, è mutilo delle parti iniziali e finali sia della colonna sia dei versi. Sono vergate sul recto $(\rightarrow) 16$ linee di testo, che recano una media di sei lettere ciascuna, troppo poche per poter ricostruirne con esattezza il contenuto. Della 1. 8 si conservano solo alcune tracce, che non consentono la trascrizione di alcuna lettera, mentre la 1.17 non presenta tracce di inchiostro, per cui si potrebbe soltanto supporre che fosse in eisthesis, similmente a $P$ Oxy. 2246 1l. 2-9, 33-37.

La porzione sinistra della colonna caduta in lacuna non deve esser molta, dal momento che alla 1. 13 è possibile integrare la parola con una lettera per intero (cfr. a) e la seconda è per metà visibile (cfr. y); inoltre, si scorge al di sotto dello stichos il segno di paragraphos, un ulteriore elemento che convalida l'assenza in incipit di 2-3 lettere ca.

La scrittura è una maiuscola rotonda di II secolo d.C., dai tratti abbastanza eleganti e curati, riconoscibile come propria della mano dello scriba \#A3 ${ }^{1}$ per le caratteristiche in comune con PSI 1208, quali beta in due tempi con i tratti curvi pieni, my con la pancia tonda che tocca la linea inferiore di scrittura, sigma in due tempi col tratto superiore lievemente retto, chi con il primo tratto discendente più spesso del secondo. Caratterizzante sembra essere epsilon, il cui tratto superiore orizzontale si chiude verso il basso, toccando spesso il tratto mediano (vd. 11. 9, 10, 11).

Sono visibili segni di accenti alle 1l. 4, 9, 14; un segno di allungamento sulla vocale a alla 1.7 e una paragraphos alla 1.13 parzialmente in lacuna. Quest'ultima fa propendere per la possibilità che si tratti di un dialogo, come è desumibile in P.Oxy. 2246. Stando

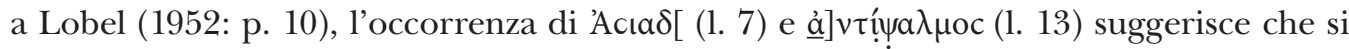

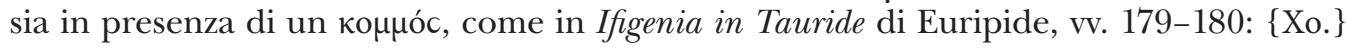

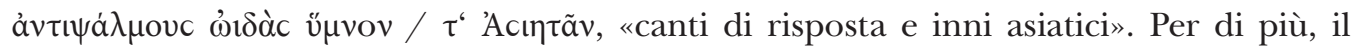
lemma $\underline{\alpha}] v \tau i ́ ̣ \alpha \lambda \mu o c(1.13)$, in incipit di verso, elimina la possibilità che si tratti di trimetri giambici. Per Dodds (ap. Snell 1953), infatti, le linee di testo sarebbero i resti di un metro bacchico.

Di seguito, offro per chiarezza una trascrizione diplomatica con apparato critico: ${ }^{2}$

\begin{tabular}{|c|}
\hline \multirow{2}{*}{ 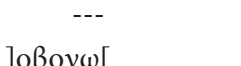 } \\
\hline \\
\hline ] $\pi v \varepsilon v \mu \alpha[][c \chi \alpha \lambda \varepsilon ı .[$ \\
\hline 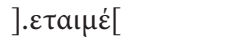 \\
\hline 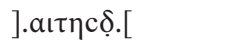 \\
\hline ].otcǫ̣̣[ \\
\hline$] a \operatorname{cācıa\delta }[$ \\
\hline ]...... [ \\
\hline 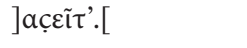 \\
\hline 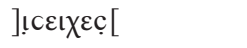 \\
\hline$] \delta \varepsilon \varepsilon \chi \chi \alpha \lambda[$ \\
\hline$] \omega \mu o . \xi \xi \varepsilon .[$ \\
\hline
\end{tabular}

1 Cfr. Johnson (2004: p. 19).

2 Il papiro è edito in Radt (1985) come fr.**451e. 


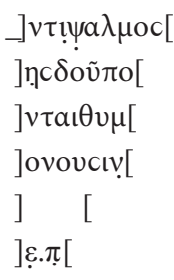

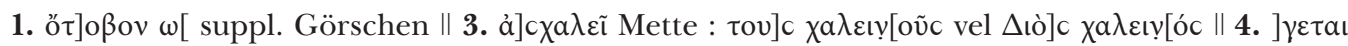

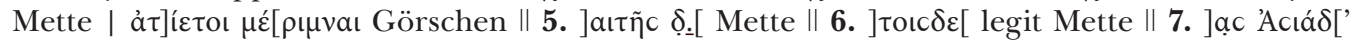

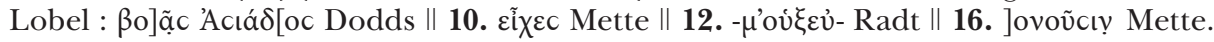

La scrittura e il layout del frammento ricordano, per Lobel (1952: p. 10), quella di P.Oxy. $2164,{ }^{3}$ e non esclude che i due frustuli derivino dal medesimo rotolo. Pertanto, ritiene plausibile che anche P.Oxy. 2248, ${ }^{4}$ come P.Oxy. 2164, fosse parte della Semele di Eschilo. ${ }^{5}$ Questa attribuzione potrebbe trovare un'ulteriore conferma nell'eventualità in cui i versi attestino lo sconvolgimento di un equilibrio naturale, dovuto all'intervento di Zeus, che nella trama della Semele seduce la figlia di Cadmo, la quale, desiderando vedere il padre degli dèi in tutto il suo splendore, ne viene folgorata. In linea con questa ipotesi, i versi sarebbero da collocare nel momento in cui Semele è stata sedotta dal dio e il Coro, comprendendo la pericolosità della situazione, ne preannuncia la morte; oppure sarebbero da inserire nel momento in cui la donna è già stata folgorata e il Coro intona un triste canto funebre.

Recuperando gli elementi dell'analogia con la mise en page di P.Oxy. 2164, Radt (1985: p. 463) ipotizza che il nostro papiro sia parte delle Xantriai, alle quali, a suo parere, apparterrebbe P.Oxy. 2164. ${ }^{6}$ Tuttavia, valutare il solo dato paleografico mi sembra argomento insufficiente, dal momento che le somiglianze grafiche e bibliologiche con altri papiri eschilei di \#A3 sono molte (cfr. e.g. PSI 1209; P.Oxy. 2249, 2253), sicché il nostro frammento potrebbe essere parte di un altro rotolo e, quindi, di un dramma diverso da

3 Il papiro è costituito da tre frammenti: i frr. 2 e 3 conservano miseri resti, rispettivamente, di nove e sette linee (nel fr. 2 si leggono i nomi di Zeus e Cadmo), laddove il fr. 1, più consistente, si articola in una sezione con cola lirici scritti in eisthesis, resti di un canto corale (vv. 1-15), seguita da esametri dattilici lirici (vv. 16-30), i primi due dei quali (1617) convergono con quanto le fonti indirette riferiscono a proposito del travestimento di Era (Plat. R. 381c-e, Diog. Sin. Ep. 34.1-2) e dell'assegnazione a Eschilo (Paus. 8.6.6) e in particolare alle sue Xantriai. È innegabile, come sottolinea Totaro (2017: p. 23), che il fr. 1, per quel che si riesce a ricostruire, abbia un contenuto dionisiaco: infatti, nella parte corale (vv. 1-15) ricorrono i nomi di Era (v. 3), Semele (vv. 9, 13), Cadmo (v. 13) e Zeus (v. 15). Dal v. 16 interviene direttamente Era che pare alludere a questioni nuziali, forse proprio al rapporto fra Semele e Zeus, dal quale nascerà Dioniso. Per un commento al papiro, dibattuto nell'attribuzione fra Semele e Xantriai, cfr. Cantarella (1942: p. 77); Galiano (1945: pp. 144-145); Giabbani (1947: p. 18, n. 255); Cantarella (1948: p. 137); Lasserre (1949: pp. 146-147); Lloyd-Jones (1957: pp. 566-571); Wartelle (1971: p. 322, n. 3); Hutchinson (1985: p. XIVI, n. 28); Diggle (1998: pp. 22-23); e il recente contributo di Totaro (2017: pp. 17-34).

4 L'ipotesi è accolta anche da Görschen (1955: p. 201; 1961: p. 107).

5 Una ricostruzione della trama della Semele è in Lucas De Dios (2008: pp. 595-599); per un'analisi dei frammenti ad essa riferiti cfr. Radt (1985: pp. 335-336).

6 Cfr. Radt (1985: pp. 281-286). Per un'indicazione della trama vd. Lucas De Dios (2008: pp. 486-488). 
uno della Licurgia. In generale, si deve notare che la dimensione dei moduli non aiuta nell'attribuzione di un frammento al medesimo papiro, poiché tendenzialmente l'altezza delle lettere $(0.4-0.5 \mathrm{~cm})$ rimane costante nella maggior parte dei frustuli a noi pervenuti; così come il colore e la lavorazione del supporto materiale non differiscono molto da un papiro all'altro: le uniche differenze sono causate dalla modalità di conservazione del lacerto prima del rinvenimento archeologico.

Analizziamo ciò che si può ricavare dal frammento.

Görschen (1955: p. 201) propone al v. 1 ö $]$ oßov, accolto anche da Mette (1959): è l'ac-

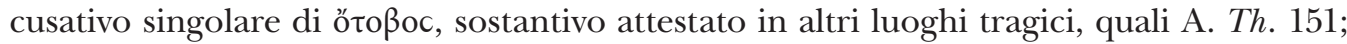
S. Aj. 1201, OC 1479; fr. 247.1 Kannicht \& Snell. Il lemma è una sorta di iperonimo del rumore, poiché designa l'idea di un suono rumoroso, prodotto da elementi naturali, quali il mare o il tuono, ovvero da un esercito che muove in battaglia (cfr. LSJ s.v.; DELG s.v. II). Bisogna, infine, evidenziare come in Eschilo l'arco di occorrenza del sostantivo őtoßoc sia totalmente coperto dal testo dei Sette contro Tebe (vd. vv. 204-205, 151).

La congettura formulata da Görschen sembrerebbe trovare plausibili riscontri in entrambe le eccezioni: in prima istanza, qualora dovesse riferirsi a eventi naturali, si potrebbe trovare un'associazione in ] $\pi v \varepsilon v \mu a ̣[$ al v. 2. Questa lectio richiama senza esitazione l'elemento del soffio e del vento (cfr. P.Oxy. 2246.22), spesso connotazione nei versi eschilei di eventi infausti (cfr. Pennesi 2008: p. 37, n. 123). Se così fosse, si potrebbe ipotizzare che ci sia un qualche riferimento al mare in tempesta, metafora di situazioni perigliose (cfr. e.g. PSI 1208.3; A. Th. 62-64, 443; E. Supp. 473-475, Ph. 859-861). L'analogia marina è largamente diffusa nella poesia tragica, e nella letteratura greca in generale, dove denota argomenti differenti, dall'amore tormentato nei confronti di un amasio $^{7}$ al momento di pericolo in cui versa la polis a causa di staseis interne. ${ }^{8}$ Non mancano, quindi, similitudini ambientali che effigiano situazioni dove le onde marine sono i corpi armati e i soldati, mentre il vento tempestoso è la furia impellente di Ares (cfr. e.g. A. Th. 114-115). In questo modo, ci si avvicina a ö $\tau$ ]oßov di Hes. Th. 709, in cui «frastuono» è riferito a un esercito in battaglia. Giova, in verità, segnalare che il sostantivo può indicare semplicemente un suono fragoroso, per cui vd. e.g. A. Th. 151, FGrH 203.3b.2, Et. M. s.v. o 636.55 [ed. Gaisford]; ovvero una dolce melodia di zampogna, per cui vd. e.g. A. $P V$ 574-575.

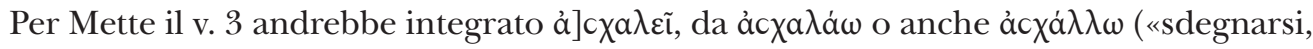
pentirsi»). Il verbo ricorre al futuro soltanto nel Prometeo incatenato di Eschilo, al v. 764,

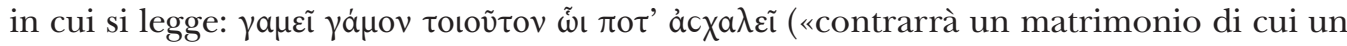
giorno si pentirà»); mentre altrove è attestato il presente, per cui cfr. e.g. H. Od. II.193, S. OT 937, E. Or. 785, IA 920.

7 Cfr. e.g. Sapph. fr. 47 Voigt; Ibyc. fr. 286.8-13 Davies; Men. fr. 420 Kassel \& Austin; Mel. AP 12.53 (= HE 4328-4335), Charit. 3.2.6; Nonn. D. 42.58-59; Cercid. 5.1-17 Powell; Aristaen. Ep. 1.10.49-51, 1.17.30-32, 1.27.43-44. Per indicazioni bibliografiche sull'argomento cfr. Kost (1971: pp. 408-410); Mastromarco (1998: pp. 115-116); Mastromarco (2000: p. 465, n. 24).

8 Cfr. e.g. Alc. fr. 208a Voigt, Theogn. 667-682; A. Th. 208-210, 759-761, Suppl. 345. Per uno studio sulla metafora politica del mare e della nave cfr. Degani \& Burzacchini (1977: p. 219), Cavallini (1986: pp. 78-81), Gerber (1997), Rodríguez Somolinos (1998: p. 98). 
Pur trovandoci in un contesto lacunoso, un'ulteriore ipotesi di lettura potrebbe considerare ]c come la lettera conclusiva di una parola di piccole dimensioni, e $\chi a \lambda \varepsilon \varepsilon_{\text {. }}[$ l'inizio di un'altra. A tal proposito, ritengo necessario far presente, sebbene con estrema prudenza, la possibilità che $\chi a \lambda \varepsilon$ เ. [ sia una forma verbale di $\chi \alpha \lambda$ เvó $\omega$, «imbrigliare, tenere a freno", spesso con senso metaforico; ne si ha un riscontro in P.Oxy. 2160.75: ].c $\chi \alpha \lambda\{\varepsilon\}$ เvoĩ [c, dove è presente il verbo all'ottativo. Un'ulteriore lettura potrebbe essere $\chi a \lambda \varepsilon$ เ (possibile anche in altri casi), sostantivo per $\chi a \lambda$ เvóc, attestato anche nel lessico tragico,

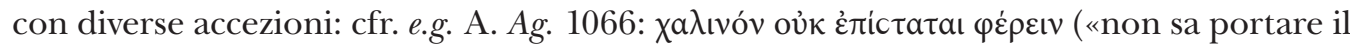

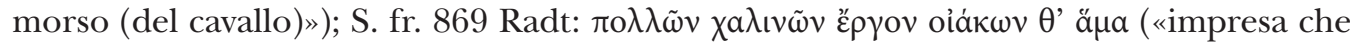

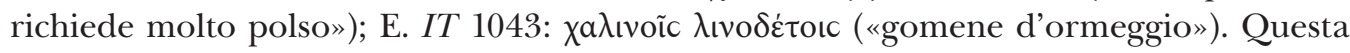
correzione del testo lacunoso troverebbe un riscontro paleografico nel segno visibile sulla linea di base, prima della lacuna e dopo lo iota: della lettera caduta in lacuna si nota un piccolo segno ascendente, lievemente inclinato, assai simile al primo tratto verticale di ny, che ha la tendenza ad esser non propriamente dritto, ma ad assumere quasi un'ornamentazione iniziale (vd. 11. 11, 16).

Fra le diverse occorrenze del lemma, interessante è la iunctura eschilea, leggibile ai vv.

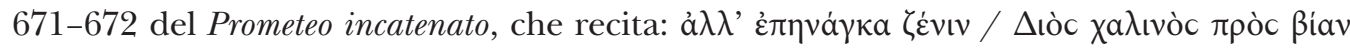
$\pi \rho a ́ c c \varepsilon ı v ~ \tau a ́ \delta \varepsilon$, «la briglia di Zeus lo costringeva con la violenza ad agire così», da intendere come la volontà forte e vincolante del padre degli dèi, in grado di frenare e guidare le decisioni di chiunque (qui, di Io, personaggio parlante). Il parallelo del nesso incipitario $\Delta$ ı̀c $\chi$ a $\lambda$ ıvóc al v. 672 del Prometeo incatenato, con il terzo verso di P.Oxy. 2248, seppur lacunoso, mi sembra significativo e non trascurabile. L'incipit sarebbe, di conseguenza, così formulato: $\Delta$ iò]c $\chi \alpha \lambda \varepsilon ı$ [óc. Per quanto riguarda la prima voce bisogna asserire che potrebbe rientrare negli spazi: la porzione sinistra del testo, infatti, parrebbe avere subito la perdita di due o tre moduli (vd. supra).

$\mathrm{Al}$ di là del sintagma $\Delta \mathrm{iò}] \mathrm{c} \chi \alpha \lambda \varepsilon ı$ [ $[$ óc, si può supporre che il sostantivo fosse preceduto

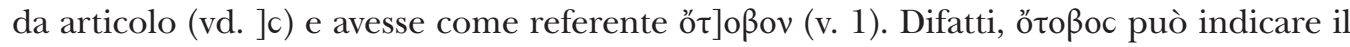

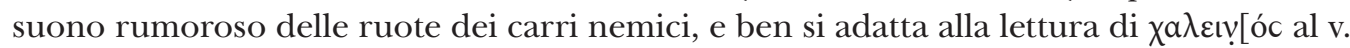
3. L'uso dei due termini, per giunta non molto distanti fra loro, potrebbe, dunque, essere concettualmente adeguato. A tal proposito, rinvio ai vv. 203-207 dei Sette contro Tebe:

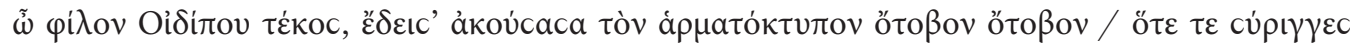

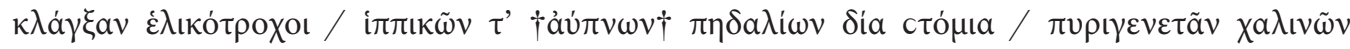
( «o caro figlio di Edipo, ti sei spaventato / nell'udire il rumore, rumore roboante dei carri, / e ciò che stridettero i mozzi che fanno girare le ruote, / e gli insonni timoni equini

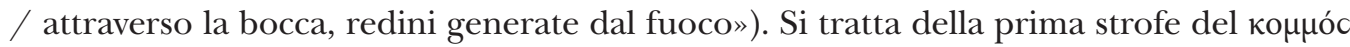
del Coro, che esprime il proprio terrore all'avvicinarsi dei nemici: si noti un'insistenza delle ripetizioni e un'amplificazione degli effetti fonici, modulati sul tono agitato dei docmi, adoperati per rappresentare il moto continuo di paura e angoscia. ${ }^{9}$

L'analogia semantica fra i due passi, potrebbe agevolare l'ipotesi dell'appartenenza

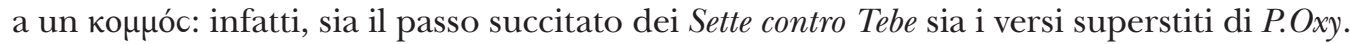
2248 presentano un assetto ritmico di natura bacchica, che sembra essere frequente nel-

9 Per un commento puntuale ai versi vd. Novelli (2005: pp. 111-130). 
le porzioni meliche corali di Eschilo contrassegnate da una forte emotività, come ai vv. 218-227 e 228-238 dell'Agamennone.

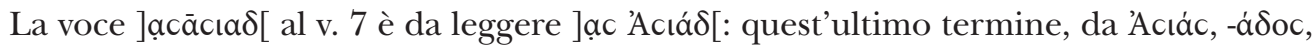
è generico e raro, ma occorre, spesso per ragioni metriche, in Eschilo ed Euripide, anche nella forma Acíc, -íoc: cfr. A. Pers. 61, 270, PV 735, Supp. 547; E. Or. 1397, IT 178-185, 396-397, An. 1, 119, Herc. 643-644, Tr. 1219, Ba. 1168. È usato di frequente in contesti negativi a indicare, soprattutto, il contrasto fra Europa e Asia, fra Occidente ed Oriente, sia in modo esplicito sia implicito, per suggerire l'estraneità dei modi, dei costumi e delle origini della persona di cui si sta parlando. Fra le ipotesi integrative Dodds (ap. Snell) propone $\beta o]$ ãc Acciá $\delta[o c$, supponendo che -ac sia un genitivo; difficile, invece,

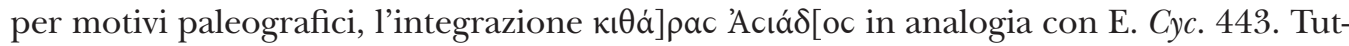
tavia, considerato il contesto, si dovrebbe individuare qualcosa di senso affine che rinvii al mondo del canto e della musica. Resta, invece, dubbio il caso del lemma Aciác.

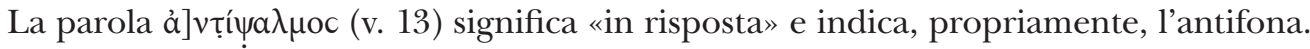

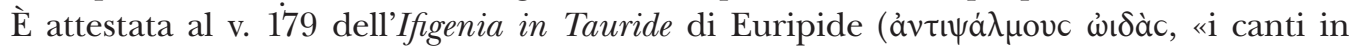
risposta»), ${ }^{10}$ dove il Coro di donne, pronuncia un canto in risposta (vv. 179-202) alle parole di Ifigenia (vv. 145-177), accompagnando con i lamenti le sofferenze della famiglia della giovane e partecipando emotivamente al dolore che l'ha colpita, in un susseguirsi sempre più concitato di suoni, rimandi, espressioni di commiserazione. Di particolare

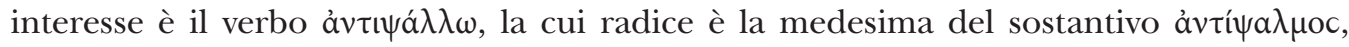
che individua propriamente l' «accompagnamento di un canto con la lira». Tale imma-

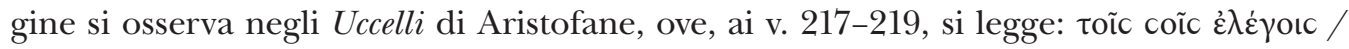

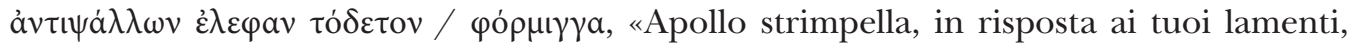
la lira». Riguardo a ciò, gli scholia vetera ${ }^{11}$ al verso confermano l'evidenza che il verbo dichiara propriamente l'uso, durante il canto, della kitharis, termine interscambiabile con lyra.$^{12}$ Pertanto, non è fuori luogo credere che anche al v. 13 di P.Oxy. 2248 si alluda, forse, a un canto di lamento con accompagnamento strumentale.

Si deve aggiungere che ả] $v \tau$ í $\psi \alpha \lambda \mu o c$ si armonizza con 'Acı́́ $\delta[-$ al v. 7, ancora in affinità con i vv. 179-180 dell'Ifigenia in Tauride euripidea: qui, il canto di risposta del Coro è

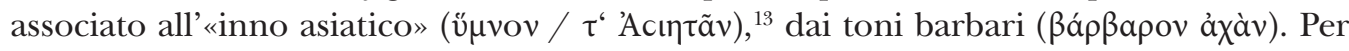
la pratica comune in Oriente del lamento appassionato, pieno di artifici, urla strazianti e gesti eclatanti: cfr. e.g. A. Pers. 135, Ch. 423-424, E. Or. 1395-1397, Ph. 1301-1302. In particolare, si può citare una sezione dei Persiani di Eschilo (vv. 935-947), ${ }^{14}$ allorché il Coro innalza un threnos, incitato dai toni patetici delle parole di Serse, che denuncia il suo infelice destino per mano di un dio demone (vv. 942-943). Qui, il Coro di Persiani lancia delle grida (vv. 935, 947), pronuncia olooĩ (v. 955), versa molte lacrime (v. 940), mentre viene portato alla memoria il momento della disfatta persiana sulla «spiaggia

10 Vd. anche supra.

11 Cfr. Evet. ad Ar. Av. 218a (p. 39 Holwerda).

12 Cfr. il commento al v. 28 di P.Oxy. 2246.

13 Sulle caratteristiche asiatiche della musica greca vd. West (1992: p. 388).

14 Per un commento al testo eschileo vd. Belloni (1988: pp. 237-239). 
funesta» (v. 965) di Salamina. Ed è proprio Serse a suggerire la modalità del lamento

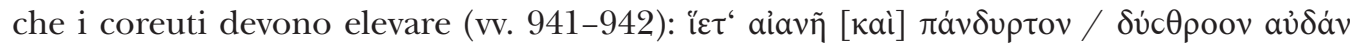
(«emettete una voce che molto geme, senza tregua, tristo suono»). Sono queste, dunque, le caratteristiche del canto asiatico, la cui ripresa è nei versi del Coro dell'Ifigenia in Tauride, che canta non più un peana, ma un inno caro ad Ade (vv. 184-185).

La presenza del segno di paragraphos in incipit di verso segnala probabilmente un cambio di interlocutore, ma è difficile, vista la frammentarietà del supporto, comprendere se sia dovuto a un passaggio che registra il dialogo fra personaggio e Coro oppure fra corifeo e Coro.

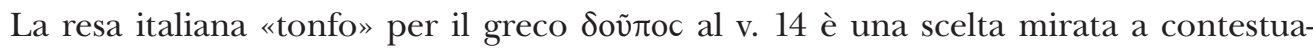

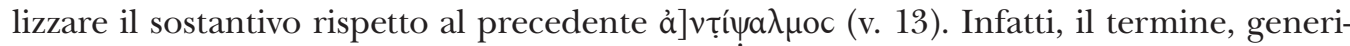
camente inteso per «rumore», trova un significativo parallelo ai vv. 632-633 dell'Aiace

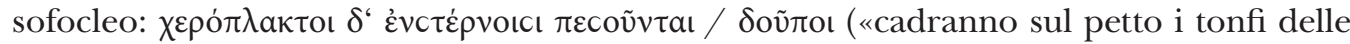
mani»). I versi descrivono la reazione della madre di Aiace, oramai anziana, che, una volta appresa la notizia della follia del figlio, non farà altro che strapparsi i capelli ingrigiti

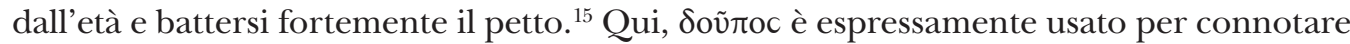
il tipo di rumore che provocano i colpi inferti al petto durante la ritualità del lamento. ${ }^{16}$ Battersi il petto e strapparsi i capelli, infatti, sono elementi tradizionali del lamento, specialmente funebre, e si accompagnano al pianto, a volte sostenuto dal canto. ${ }^{17}$ Numerosi sono i confronti ${ }^{18}$ rintracciabili in altri passi tragici, quali e.g. A. Ch. 22-31, 423-428; S. Aj. 310, El. 88-90; E. An. 826-827, 1209 - si badi che alcuni di questi versi fanno riferimento anche al canto asiatico, per cui vd. supra. ${ }^{19}$

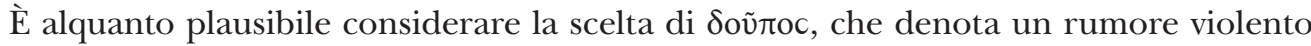
e cupo, come necessità scenica di fornire un'indicazione performativa al pubblico. Da qui, l'idea secondo cui gli spettatori, seduti nelle file più lontane del teatro, non fossero in grado di percepire il rumore delle percosse che il Coro si infliggeva e, di conseguenza, fosse necessario fornire un'indicazione didascalica sulla tipologia di rumore provocato da un gesto simile; inoltre il sostantivo suggerisce in modo concreto l'immagine del dolore, poiché la violenza che viene auto-provocata è esternazione del sentimento interiore, che è davvero straziante.

Pertanto, dal punto di vista contenutistico, si può ritenere che $\delta$ oṽ $\pi$ [ ben si coniughi

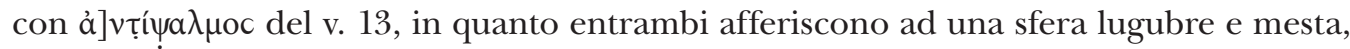
legata alla ritualità del canto e dei gesti, motivi tipici del lamento, ${ }^{20}$ in grado di esprimere sulla scena sentimenti cupi e gravi provocati da infelici notizie, quali la sconfitta in una

15 Si discute se questi passaggi siano «improvised laments at a special directly inspired by the sorrow of the occasion» oppure «formal lamentations at a special ceremony» (Lloyd-Jones 1985: p. 17).

16 Per uno studio sulla gestualità e sulla modalità con cui veniva espresso il dolore nel mondo greco cfr. Alexiou (2003).

17 Sulla ritualità del threnos in tragedia vd. le indicazioni fornite da Garvie (1986: pp. 54-55).

18 Cfr. P.Oxy. 2251.7.

19 Per ulteriori esempi in tragedia e in altri luoghi della letteratura greca rinvio a Finglass (2011: p. 324).

20 Su questo motivo in tragedia vd. Vickers (1973: pp. 88-90). 
guerra (cfr. A. Pers. 941-943), la devastazione di una famiglia (cfr. A. Ch. 424-428), la morte di una persona cara (cfr. S. Aj. 631-634).

Dai dati sin qui raccolti, si può desumere che il frammento papiraceo è certamente una testimonianza diretta di un testo tragico, di cui non restano che pochi stralci di un dialogo fra il Coro e forse un coreuta o un altro personaggio. È difficile rilevare se i versi siano per davvero eschilei, sicché il confronto paleografico della mano dello scriba con altri lacerti attribuiti al tragediografo resta l'unico dato (verosimile) in nostro possesso, dal momento che né il contenuto ricostruibile dai pochi versi né, tantomeno, lo studio lessicale dei lemmi leggibili concorrono ad assegnare con assoluta sicurezza P.Oxy. 2248 a Eschilo. Neppure l'analisi contenutistica e i confronti con altri luoghi tragici aiutano a trarre delle plausibili conclusioni, dal momento che si trovano analogie sia con Sofocle sia con Euripide. Infatti, i versi ricostruibili alludono a situazioni tragiche generiche, che non bastano ad assicurare, senza esitazione, la paternità eschilea di P.Oxy. 2248.

I versi descrivono, con ogni probabilità, una situazione di pericolo imminente o già passato, nella quale si trovano il Coro ovvero gli altri personaggi del dramma. I vv. 1-3 potrebbero, quindi, accennare a dei rumori dovuti all'imperversare di una guerra o di una battaglia, che provoca un tumulto simile ad una tempesta (v. 2), alla quale non è possibile scampare, poiché ineludibile e violenta, e forse condotta dal volere di un dio (v. 3: $\Delta$ iò]c $\chi a \lambda \varepsilon ı$ [̣́c ?). Tuttavia, il presunto scontro potrebbe anche essersi già concluso, lasciando dietro di sé soltanto morte, devastazione e disperazione. Di conseguenza, il Coro (v. 13) compiangerebbe la propria sorte oppure di quanti vivono, o hanno vissuto, la drammaticità dell'evento, innalzando un inno dai toni sinistri e funerei, accompagnati da gesti violenti (v. 14) che denunciano la profondità del dolore.

Tale interpretazione è, però, soltanto una possibilità. Infatti, i vv. 1-3 potrebbero far riferimento a una situazione per nulla ascrivibile a un evento bellico, ma evocherebbero un momento di particolare terrore e disperazione dovuto a cause di tutt'altra natura, come una situazione di capovolgimento nefasto del destino dell'interlocutore o di un altro personaggio di cui si parla. In ogni caso, qualsiasi sia la ragione che abbia potuto condurre alla caratterizzazione di un ambiente dai tratti infausti, va evidenziato che a partire dal v. 13 vi è un richiamo a un scena di lamento e di compartecipazione al dolore.

\section{Bibliografia}

Alexiou, M. $\left(2003^{2}\right)$. The Ritual Lament in Greek Tradition. Cambridge: University Press.

Belloni, L. (Ed.). (1988). Eschilo: I Persiani. Milano: Pàtron Editore.

Cantarella, R. (1942). Nuovi frammenti papiracei di Eschilo. Dioniso, 9, 75-79.

Cantarella, R. (1948). I nuovi frammenti eschilei di Ossirinco. Napoli: Libreria Scientifica Editrice.

Cavallini, E. (1986). Presenza di Saffo e Alceo nella poesia greca fino ad Aristofane. Ferrara: Giornale filologico Ferrarese.

Degani, E., \& Burzacchini, G. (1977). Lirici greci. Firenze: Palumbo Editore.

Diggle, J. (1998). Tragicorum Graecorum fragmenta selecta. Oxford: University Press.

Finglass, P. J. (Ed.). (2011). Sophocles: Ajax. Cambridge: University Press. 
Galiano, M. F. (1945). Los papiros de tragedias griegas, en los últimos años. Investigación y Progreso, 16, 139-153.

Galiano, M. F. (1961). Les fragments d'Eschyle. In L. Amundsen, \& V. Skanland (Eds.), Proceedings of the IX International Congress of Papyrology. Oslo, 19th-22nd August, 1958 (pp. 81-133). Oslo: University Press.

Garvie, A. F. (Ed.). (1986). Aeschylus: Choephori. Oxford: University Press.

Gerber, D. F. (1997). A Companion to Greek Lyric Poets. Leiden: Brill.

Giabbani, L. (1947). Testi letterari greci di provenienza egiziana (1920-1945). Firenze: Ariani.

Görschen, F. C. (1955). Neue Fragmente aus Aischylo's 'Philoktet'. Gymnasium, 62, 200-206.

Hutchinson, G. O. (Ed.). (1985). Aeschylus: Septem contra Thebas. Oxford: University Press.

Johnson, W. A. (2004). Bookrolls and Scribes in Oxyrhynchus. Toronto: University Press.

Kannicht, R., \& Snell, B. (1981). Fragmenta adespota. Testimonia volumini 1 addenda. Indices ad volumina 1 et 2. Göttingen: Vandenhoeck \& Ruprecht.

Kassel, R., \& Austin, C. (1983-2001). Poetae Comici Graeci, voll. I-VIII. Berolini: Novi Eboraci.Kost, K. (1971). Musaios. Hero und Leander. Bonn: Bouvier Verlag Herbert Grundmann.

Lasserre, F. (1949). Les 'Xantriai' d'Eschyle. Museum Helveticum, 6, 140-156.

Lloyd-Jones, H. (1967). Appendix. In H. W. Smyth (Ed.), Aeschylus, II: Fragments (pp. 523-603). London: University Press.

Lloyd-Jones, H. (1985). Sophocles: ‘Ajax’ 624f. In C. Schäublin (Ed.), Catalepton. Festschrift für Bernard Wyss 80. Geburtstag (pp. 16-18). Basel: Seminar für klassische Philologie der Universität Basel.

Lobel, E., Roberts, C. H., \& Wegener, E. P. (1941). The Oxyrhynchus Papyri, Part XVIII. London: Egypt Exploration Society.

Lucas De Dios, J. M. (2008). Esquilo. Fragmentos - Testimonios. Introducciones, traducciòn y notas. Madrid: Editorial Gredos.

Lobel, E., Roberts, C. H., \& Wegener, E. P. (1952). The Oxyrhynchus Papyri, Part XX. London: Egypt Exploration Society.

Mastromarco, G. (1998). Scene notturne in Menandro e Turpilio. Seminari Romani di cultura greca, 1, 111-121.

Mastromarco, G. (2000). Scene notturne nella commedia greca. In M. Cannatà Fera, \& S. Grandolini (Eds.), Poesia e religione in Grecia. Studi in onore di G. Aurelio Privitera (Vol. 2; pp. 457-468). Napoli: Edizioni scientifiche italiane.

Mette, H. J. (1959). Die Fragmente der Tragödien des Aischylos. Berlin: Akademie-Verlag.

Novelli, S. (2005). Studi sul testo dei Sette contro Tebe. Amsterdam: Adolf M. Hakkert Editore.

P.Oxy. Oxyrhynchus Online. Image Database. The Oxyrhynchus Papyri. University of Oxford [accessed 24.03.2020; http:/ / 163.1.169.40/cgi-bin/library?e=d-000-00-0POxy-00-0-0-0prompt-10-4 -0-11-1en-50-20-help-00031-001-1-0utf-00\&a=d\&c=POxy\&cl=CL1.1\&d=HASHa38fe08841bfla4ea87355].

Pennesi, A. (2008). I frammenti della 'Niobe' di Eschilo. Amsterdam: Adolf M. Hakkert Editore.

Radt, S. L. (1985). Tragicorum Graecorum Fragmenta (TrGF), III: Aeschylus. Göttingen: Vandenhoeck \& Ruprecht.

Rodríguez Somolinos, H. (1998). El Léxico de los poetas lesbios. Madrid: Consejo Superior de Investigaciones Científicas.

Snell, B. (1953). Rec. a Lobel 1952. Gnomon, 25, 433-440. 
Totaro, P. (2017). Eschilo, P.Oxy. 2164, fr. 1, 16-17. In F. Conti Bizzarro, G. Massimilla, \& G. Matino (Eds.), PHILOI LOGOI. Giornate di studio su Antico, Tardoantico e Bizantino dedicate ad Ugo Criscuolo (pp. 17-34). Napoli: Satura Editrice.

Vickers, B. (1973). Towards Greek Tragedy: Drama, Myth, Society. London - New York: Longman.

Wartelle, A. (1971). Histoire du texte d'Eschyle dans l'antiquité. Paris: Société d'Édition Les Belles Lettres.

West, M. L. (1992). Ancient Greek Music. Oxford: University Press.

Daniela Immacolata Cagnazzo / daniela.cagnazzo@uniba.it

PhD Student in Classics

Department of Humanities

University of Bari Aldo Moro

Palazzo Ateneo - Piano 2 , Piazza Umberto I, 1, 70121 Bari, Italy 